\title{
Regular Tunnelling Sequences in Mixed Systems
}

\author{
Stephen C. Creagh ${ }^{\mathrm{a}}$ and Niall D. Whelan ${ }^{\mathrm{b}}$ \\ ${ }^{a}$ Division of Theoretical Mechanics, School of Mathematical Sciences \\ University of Nottingham, NG7 2RD, UK
}

${ }^{\mathrm{b}}$ Department of Physics and Astronomy, McMaster University, Hamilton, Ontario, Canada L8P 2E7

\begin{abstract}
We show that the pattern of tunnelling rates can display a vivid and regular pattern when the classical dynamics is of mixed chaotic/regular type. We consider the situation in which the dominant tunnelling route connects to a stable periodic orbit and this orbit is surrounded by a regular island which supports a number of quantum states. We derive an explicit semiclassical expression for the positions and tunnelling rates of these states by use of a complexified trace formula.
\end{abstract}

PACS: 03.65.Sq, 73.40Gk, 05.45.Mt, 05.45.-a

Keywords: tunnelling, chaos, periodic orbit theory

Tunnelling in systems whose classical limit displays a mixture of chaotic and integrable behaviour 1 , 4 is often quite complex and impossible to predict analytically. Much attention has been paid recently, for example, to the regime of chaos-assisted tunnelling [2] in which dynamical tunnelling occurs between quasimodes supported in integrable island-chains embedded in a chaotic sea. By contrast, we report on a remarkably ordered structure that appears in the tunnelling behaviour of a particular kind of mixed system and give analytical estimates for the corresponding tunnelling rates. It is distinct from the case of chaos-assisted tunnelling because tunnelling is through an energetic barrier rather than through dynamical barriers such as KAM tori. The special feature of these systems is that the complex orbit which defines the optimal tunnelling route across the barrier connects to a stable periodic orbit at the centre of an island chain (which is generally embedded in a chaotic sea).

The ordered nature of these tunnelling rates is immediately evident in Fig. 1 1 where we show the numerically obtained splittings between quasidoublets of the double-well potential $V(x, y)=$ $\left(x^{2}-1\right)^{4}+x^{2} y^{2}+2 y^{2} / 5$. We have held the en-

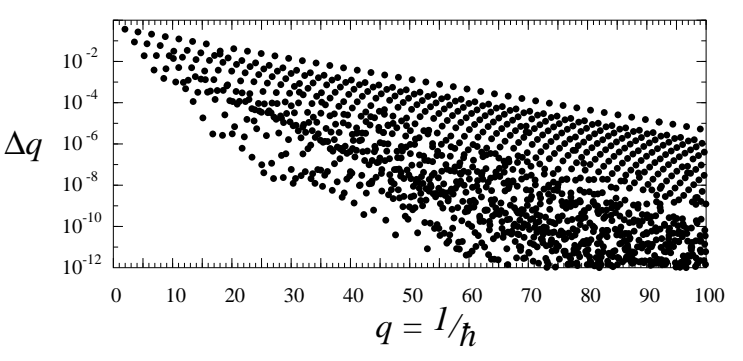

Figure 1. Each dot represents a splitting doublet. The horizontal coordinate is the mean value of $q=$ $1 / \hbar$ and the vertical coordinate is the splitting.

ergy fixed (at $E=9 / 10$ ) and found the values of $q=1 / \hbar$ for which this is an energy level. The resulting spectrum of $q$ values is equivalent in most respects to a standard energy-spectrum, with the advantage that the classical dynamics is fixed throughout.

The largest splittings in Fig. 1 are highly ordered, forming a regular progression of families which grow larger in number higher in the spectrum. These correspond to states supported near the centre of the island and we will offer a simple analytical prediction for them. The smallest splittings in Fig. 1 form a disordered jum- 
ble. These correspond to states supported in the chaotic sea and dynamically excluded from the main tunnelling route.

To analyse the ordered sequence we use a method developed in [5.6] and used until now primarily to understand predominantly chaotic systems. We first present the analysis for the case that $q=1 / \hbar$ is held fixed and an energy-spectrum is computed. We then give the simple extension for the fixed energy $q$-spectrum, appropriate for the results of Fig. 11. We call the mean energy of the $n$ 'th doublet $E_{n}$ and the corresponding splitting $\Delta E_{n}$ and define the following dimensionless spectral function

$f(E, q)=\sum_{n} \Delta E_{n} \delta\left(E-E_{n}\right)$.

There is an analogous definition for metastable wells which have extremely narrow resonances, in which the widths $\Gamma_{n}$ play the role of splittings. Such a system was studied in [7] for a fully integrable system; the structure of the spectrum was like that shown here except with no irregular jumble at the bottom. While similar in outline, the detailed method of analysis was rather different, making use of the action-angle variables which exist in that situation.

In [5.6] we approximate (11) semiclassically as a sum over complex tunnelling orbits which traverse the barrier (in analogy to Gutzwiller's formula for the density of states using real orbits [8]). We shall consider the special case in which there is an additional reflection symmetry such that the dominant tunnelling route lies on the symmetry axis so that it connects smoothly to a real periodic orbit. We then identify three distinct contributions to $f(E, q)$. There is the so-called instanton which has a purely imaginary action $i K_{0}$, lives under the barrier and runs along the symmetry axis between the classical turning points. It is important for determining the mean behaviour of the splittings but does not affect the fluctuation effects which we are trying to capture here. The second contribution comes from orbits which execute real dynamics along the real periodic orbit lying on the symmetry axis in addition to the instanton dynamics beneath the well. We imagine an orbit which starts at one of the turning points, executes $r$ repetitions of the real periodic orbit and then tunnels along the instanton path to finish at the other turning point. This orbit has a complex action $S=r S_{0}+i K_{0}$ where $S_{0}$ is the real action of the real periodic orbit. The contribution to $f(E, q)$ is given by [5]

$f_{\mathrm{osc}}(E, q)=\frac{2}{\pi} \operatorname{Re} \sum_{r=1}^{\infty} \frac{e^{-q K_{0}+r i q S_{0}}}{\sqrt{-\operatorname{det}\left(W_{0} M_{0}^{r}-I\right)}}$.

The matrices $W_{0}$ and $M_{0}$ are the monodromy matrices of the instanton and of the real periodic orbit respectively; the composite orbit has a monodromy matrix which is simply a product of these. The third contribution, discussed in [6], comes from homoclinic orbits which explore the real wells far away from the symmetry axis. They play no role in the present discussion.

For fully developed chaos, all periodic orbits are unstable. The denominator of (2) then decays exponentially with $r$ and large repetitions are numerically unimportant. If the orbit is stable, however, there is no corresponding suppression. Singularities corresponding to distinct states arise when the expression is summed. This follows very closely the analogous development of Miller and Voros 911 for the Gutzwiller trace formula when there is a stable orbit, except here we find splittings in addition to the positions of energies. (As was done in one dimension by Miller [12].)

In the stable case, $M_{0}$ has eigenvalues $e^{ \pm i \alpha}$ on the unit circle. (In higher dimensions, there would be a number of such eigenvalues and the theory would be generalised accordingly.) Let the diagonal matrix elements of $W_{0}$ in the complex eigenbasis of $M_{0}$ be $A$ and $B$ so that

$$
\begin{aligned}
-\operatorname{det}\left(W_{0} M_{0}^{r}-I\right) & =\operatorname{Tr} W_{0} M_{0}^{r}-2 \\
& =A e^{i r \alpha}+B e^{-i r \alpha}-2 .
\end{aligned}
$$

Since the instanton's period is imaginary, complex conjugation acts as a time-reversal operation and we find that $W_{0}^{*}=W_{0}^{-1}$. We therefore conclude that $\left(\operatorname{Tr} W_{0} M_{0}^{r}\right)^{*}=\operatorname{Tr}\left(W_{0} M_{0}^{-r}\right)^{-1}=$ $\operatorname{Tr} W_{0} M_{0}^{-r}$ (the latter equality holds because every symplectic matrix is conjugate to its inverse). Comparing this with (3) we conclude that $A$ and $B$ are real; we discuss how they are computed and offer a geometrical interpretation in the appendix. 
We now make use of the generating function of the Legendre polynomials to conclude

$$
\frac{1}{\sqrt{-\operatorname{det}\left(W M_{0}^{r}-I\right)}}=\sum_{k=0}^{\infty} e^{i(k+1 / 2) r \alpha} \frac{Q_{k}(A B)}{B^{k+1 / 2}}(4)
$$

where we assume without loss of generality that $B$ is the larger in magnitude of $(A, B)$ and we let $Q_{k}(z)$ denote the polynomial

$Q_{k}(z)=z^{k / 2} P_{k}\left(z^{-1 / 2}\right)$.

Using this in (2) and summing the resulting geometric series in $r$ for each $k$ we get,

$f_{\mathrm{osc}}(E)=\frac{2 e^{-q K_{0}}}{\pi} \operatorname{Re} \sum_{k=0}^{\infty} \frac{a_{k}}{e^{-i \Phi_{k}}-1}$,

where we have defined

$$
\begin{aligned}
a_{k} & =\frac{Q_{k}(A B)}{B^{k+1 / 2}} \\
\Phi_{k} & =q S_{0}+(k+1 / 2) \alpha .
\end{aligned}
$$

Semiclassical energy levels are found when the distribution above has poles and are implicit solutions $E_{m k}$ of $\Phi_{k}=2 \pi m$. From the residues we recover estimates of the corresponding splittings. This is a form of torus quantisation in which $k$ is a transverse quantum number, treated in harmonic approximation, and $m$ counts nodes along the orbit. The corresponding states are localised on the tori surrounding the stable periodic orbit and we find that their respective tunnelling rates are much larger than those of other states.

Note that near $E_{m k}$ we can write

$$
\frac{1}{e^{-i \Phi_{k}}-1} \approx \frac{i}{q T_{0}} \frac{1}{E-E_{m k}},
$$

where we have used that the period is $T_{0}=$ $\partial S_{0} / \partial E$. Using the standard identity

$\operatorname{Im} \frac{1}{E-E_{m k}}=-\pi \delta\left(E-E_{m k}\right)$

we conclude

$\Delta E_{m k}=\frac{2 \hbar}{T_{0}} e^{-K_{0} / \hbar} a_{k}\left(W_{0}, M_{0}\right)$,

where the notation now stresses that $a_{k}$ depends on the transverse dynamics of the instanton and its real extension through the monodromy matrices $\left(W_{0}, M_{0}\right)$. All classical quantities are evaluated at energy $E_{m k}$. This formula has the same form as in one-dimensional tunnelling [12] except for the additional factor $a_{k}\left(W_{0}, M_{0}\right)$. We will find this factor to be of order unity when $k$ is small and to decrease as $k$ increases.

In [5] we reported some specific numerical results for the energy quantisation. We now extend this result to the the $q$-spectrum, the advantage being that the classical quantities $K_{0}, W_{0}$ and $M_{0}$ are constant. The function $f(E, q)$ can equally well be interpreted as a function of $q$ at fixed $E$ so that (6) still applies. Solving for the poles and residues as above, we conclude

$$
\begin{aligned}
q_{m k} & =(2 m \pi-(k+1 / 2) \alpha) / S_{0} \\
\Delta q_{m k} & =\frac{2}{S_{0}} e^{-q_{m k} K_{0}} a_{k}\left(W_{0}, M_{0}\right) .
\end{aligned}
$$

The sequences of Fig. 1 1 can now be interpreted in terms of the quantum numbers $m$ and $k$.

The central states with $k=0$ correspond to the largest splittings, about 35 times larger than the local average. In Fig. 1 they are the uppermost curve of points (along which $m$ varies). Keeping $m$ fixed and letting $k$ increase one gets a sequence, which appears as a left-sloping shoulder in Fig. 1, along which $q$ and $\Delta q$ decrease.

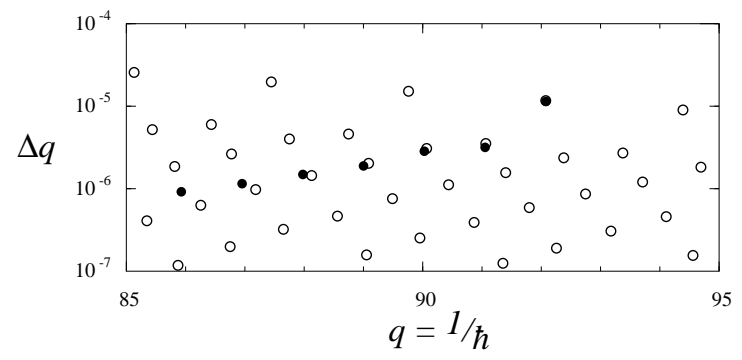

Figure 2. The open circles are numerically-computed splittings. The filled circles are the semiclassical predictions for the family with $m=40$ and $0 \leq k \leq 6$.

In Fig.2 we show a subset of the spectrum with the semiclassical predictions for $m=40$ and a sequence of $k$ values (using $A=-0.861, B=4.043$ and $\alpha=2.783$.) Clearly the small $k$ states are 
well reproduced. Our analysis essentially extrapolates the properties of the central periodic orbit to the entire island [9], and this is less accurate for the large $k$ states which are localised further from the periodic orbit. Reproducing the large $k$ values would require a more sophisticated analysis [11, although our formalism does at least capture the correct qualitative behaviour of these states. The irregular jumble of splittings at the bottom of the figure corresponds to states in the chaotic part of phase space. No simple theory exists for them though one could well imagine that the formalism of chaos-assisted tunnelling, in particular the interplay between regular and chaotic states, might be of use in describing them.

As remarked, the number of well-defined $k$ states increases as we go up in the spectrum. This is because the number of states which the regular island can support increases as $\hbar$ decreases. Also, there are occasional irregularities in the lattice of regular states, for example the $k=0$ state near $q=18$. These are due to near degeneracies between the regular state and some other state either another regular one or a chaotic one. The actual eigenstates are then strongly mixed and hence so are the tunnelling rates.

\section{Appendix}

To apply Eq. (11) in practice one needs the parameters $A$ and $B$; we give here simple basisindependent expressions for them. In particular, we note that it is not explicitly necessary to transform $W_{0}$ to the eigenbasis of $M_{0}$. We calculate $A$ and $B$ as the smaller and larger respectively of

$A$ or $B=\cosh \beta \pm \gamma \sinh \beta$

where $\operatorname{Tr} W_{0}=2 \cosh \beta$ and,

$\gamma=\frac{\operatorname{Im} \operatorname{Tr} W_{0} M_{0}}{2 \sin \alpha \sinh \beta}$.

This is obtained by expressing $W_{0}=e^{-i \beta J H}$ and $M_{0}=e^{\alpha J K}$ where $J$ is the unit symplectic matrix and $H$ and $K$ are real, positive-definite, $2 \times 2$ symmetric matrices normalised so that $\operatorname{det} H=$ $\operatorname{det} K=1$. Expanding

$W_{0}=e^{-i \beta J H}=\cosh \beta-i J H \sinh \beta$ and similarly for $M_{0}{ }^{r}=e^{r \alpha J K}$, one recovers (3) with $A$ and $B$ as given above.

The factor $\gamma$ has the following geometric interpretation. The action $K \rightarrow M K M^{T}$ of $2 \times 2$ symplectic matrices $M$ on symmetric matrices $K$ can be identified with $(2+1)$-dimensional Lorentz transformations (since the relevant Lie algebras are isomorphic), the invariant $\operatorname{det} K$ playing the role of proper time. The matrices $H$ and $K$ define unit time-like $(2+1)$-vectors $X=(x, y, t)$ and $\Xi=(\xi, \eta, \tau)$ respectively. For example

$H=\left(\begin{array}{cc}t+x & y \\ y & t-x\end{array}\right), \quad t^{2}-x^{2}-y^{2}=1$,

and similarly for $K$. One then observes that $\gamma=$ $-\operatorname{Tr} J H J K / 2=\langle X, \Xi\rangle=t \tau-x \xi-y \eta$. This can be interpreted as the dilation factor to boost the rest-frame of $X$ to that of $\Xi$. In particular, $\gamma>1$.

\section{REFERENCES}

1. W. A. Lin and L. E. Ballentine, Phys. Rev. Lett. 65, 2927 (1990)

2. O. Bohigas, S. Tomsovic and D. Ullmo, Phys. Rev. Lett. 64, 1479 (1990); Phys. Rep. 223, 45 (1993); O. Bohigas et al., Nucl. Phys. A560, 197 (1993); Tomsovic and D. Ullmo, Phys. Rev. E 50, 145 (1994)

3. F. Leyvraz and D. Ullmo, J. Phys. A 10, 2529 (1996).

4. E. Doron and S. D. Frischat, Phys. Rev. Lett. 75, 3661 (1995); Phy. Rev. E 57, 1421 (1998).

5. S. C. Creagh and N. D. Whelan, Phys. Rev. Lett. 77, 4975 (1996).

6. S. C. Creagh and N. D. Whelan, Phys. Rev. Lett. 82, 5237 (1999).

7. M. W. Beims, V. Kondratovich and J. B. Delos, Phys. Rev. Lett 81, 4537 (1998).

8. M. C. Gutzwiller, Chaos in Classical and Quantum Mechanics (Springer, 1990).

9. A. Voros, in Colloques Internationaux CNRS no. 237 ed. K. M. Souriau, pp. 277-287 (1975); Ann. Inst. H. Poincaré, A 24, 31 (1976); J. Phys. A 21, 685 (1988).

10. W. J. Miller, J. Chem. Phys 63, 996 (1975). 11. P. J. Richens, J. Phys. A 15, 2101 (1982). 12. W. J. Miller, J. Phys. Chem. 83, 960 (1979). 